Journal of

Applied

Crystallography

ISSN 1600-5767

\title{
Crystallization behavior of diblock copolymers based on PCL and PLLA biopolymers
}

\author{
Iván Navarro-Baena, Angel Marcos-Fernandez, José M. Kenny and Laura \\ Peponi
}

J. Appl. Cryst. (2014). 47, 1948-1957

Copyright (C) International Union of Crystallography

Author(s) of this paper may load this reprint on their own web site or institutional repository provided that this cover page is retained. Republication of this article or its storage in electronic databases other than as specified above is not permitted without prior permission in writing from the IUCr.

For further information see http://journals.iucr.org/services/authorrights.html

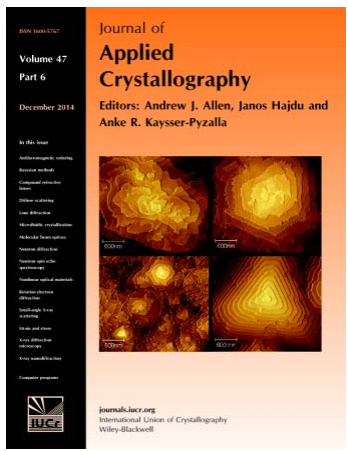

Many research topics in condensed matter research, materials science and the life sciences make use of crystallographic methods to study crystalline and non-crystalline matter with neutrons, X-rays and electrons. Articles published in the Journal of Applied Crystallography focus on these methods and their use in identifying structural and diffusioncontrolled phase transformations, structure-property relationships, structural changes of defects, interfaces and surfaces, etc. Developments of instrumentation and crystallographic apparatus, theory and interpretation, numerical analysis and other related subjects are also covered. The journal is the primary place where crystallographic computer program information is published.

\section{Crystallography Journals Online is available from journals.iucr.org}


Journal of

Applied

Crystallography

ISSN 1600-5767

Received 9 July 2014

Accepted 13 October 2014

\section{Crystallization behavior of diblock copolymers based on PCL and PLLA biopolymers}

\author{
Iván Navarro-Baena, ${ }^{a}$ Angel Marcos-Fernandez, ${ }^{b}$ José $M$. Kenny ${ }^{a, b}$ and Laura \\ Peponi $^{\text {b* }}$
}

a Department of Civil and Environmental Engineering, University of Perugia, Terni 05100, Italy, and b Institute of Polymer Science and Technology, CSIC, Madrid 28006, Spain. Correspondence e-mail: Ipeponi@ictp.csic.es

\begin{abstract}
This paper aims to increase the knowledge on the crystallinity features of diblock copolymers based on poly( $\varepsilon$-caprolactone) (PCL) and poly(L-lactic acid) (PLLA). Six diblock copolymers have been synthesized starting from a synthesized PCL with a molecular weight of around $5000 \mathrm{~g} \mathrm{~mol}^{-1}$, varying the molecular weight of the PLLA block. The crystalline unit cells for both PCL and PLLA blocks have been studied with wide-angle X-ray diffraction analysis. The effects of the copolymer composition on the crystalline cell parameters as well as on the degree of crystallinity and the crystallite sizes, determined using the Scherrer equation, are discussed. The double-crystalline nature of the diblock copolymer was confirmed by small-angle X-ray scattering experiments. This technique was also used to study the melting behavior of the copolymers by studying the variation of the diffraction spectra with temperature. The effects of PCL chains on the packing of the PLLA lamellae are discussed. Finally, the crystallization behavior was studied by differential scanning calorimetry analysis, performing experiments at different crystallization temperatures and studying the results by fitting the experimental data with an Avrami-type equation. The influence of each block on the crystallization parameters of the other block are discussed. This study allows a better understanding of the effects of the chemical structure on the crystalline behavior of these block copolymers, leading to the possibility to tailor the materials for specific applications.
\end{abstract}

Aliphatic polyesters, such as poly( $\varepsilon$-caprolactone) (PCL) and poly(L-lactic acid) (PLLA), are biopolymers commonly studied because of their extraordinary properties. In particular, PCL and PLLA are semicrystalline biodegradable polymers with different mechanical and thermal behavior but are able to offer good synergetic effects. In fact, the glass transition temperature $\left(T_{\mathrm{g}}\right)$ of PCL is around $213 \mathrm{~K}$, while for PLLA it is about $333 \mathrm{~K}$, thus indicating that PCL can reduce the rigidity of PLLA at room temperature. The crystallization and melt behavior of both polymers have been previously studied by some research groups (Loo et al., 2001; Di Lorenzo, 2005; Pan et al., 2007; Alvarez et al., 2003; Castillo et al., 2010; Sonseca et al., 2012). These authors reported that PLLA usually crystallizes in the $\alpha$ form, which consists of an orthorhombic unit cell, although different polymorphs can be obtained, such as $\alpha^{\prime}, \beta$ and $\gamma$, depending on the crystallization conditions. The melting temperature for the $\alpha$ form is around $443 \mathrm{~K}$ depending on the crystallization conditions and the molecular weight of the polymer. On the other hand, the PCL crystal unit has been reported only as an orthorhombic unit cell (Bittiger et al., 1970).

Studies on polymer crystallization have attracted the attention of many researches in recent years. In particular, 
several models able to describe the isothermal crystallization processes in polymers have been proposed. The Avrami equation is the most commonly used model for polymer crystallization, because of its good ability to interpolate the experimental results as well as its simplicity [equation (3)]. This method assumes that the growth velocity of spherulites is constant and that the nuclei are well distributed or the crystallization takes place in an infinite medium compared to the crystal size (Albano et al., 2007). However, these assumptions provoke some deviations from the real behavior. Several alternative models, generally based on the Avrami method, have been proposed. For instance, Lambrigger (1996) proposed a difference quotient method for studying the crystallization at different temperatures and to corroborate the validity of the Avrami method. Other reported methods, such as Tobin, Malkin and Urbanovici-Segal, are only simple modifications of the Avrami method (Supaphol, 2001). However, as reported by Lorenzo et al. (2007), the Avrami method fits very well in the region of 3-20\% of crystallization and, therefore, we have adopted the same approach for studying the crystallization of our materials. Moreover, the crystallization behavior of different kinds of PCL- $b$-PLLA diblock copolymers has also been reported. Kim et al. (2001) studied different PCL- $b$-PLLA diblock copolymers with different molecular weights. Their results indicated that the PLLA chain folding orientation is strongly affected by its molecular weight. They also obtained the order-disorder transition temperature at about $493 \mathrm{~K}$ by rheological measurements. Hamley et al. (2005) also studied the crystallization behavior of PCL- $b$-PLLA diblock copolymers, concluding that during the PCL crystallization there are topological restrictions that increase the crystallization times and affect the crystalline shape owing to the previous crystallization of the covalently linked PLLA block. On the other hand, the crystallization behavior is different for the PLLA block, and the presence of PCL does not affect the formation of PLLA crystals. Moreover, Hamley and co-workers reported a case in which for a $\mathrm{PCL}_{56}-b$-PLLA 44 diblock copolymer the PCL crystallization changes the already formed PLLA crystal structure (Hamley et al., 2006). Furthermore, Castillo et al. (2010) studied the Avrami exponent and the crystallization kinetics for a wide range of diblock copolymers, discovering a two-dimensional growth for PCL crystals and a three-dimensional growth for PLLA crystals. The crystallization kinetics of PCL-PLLA block copolymers have also been studied for more complex block copolymer structures, such as star-shaped copolymers (Wang \& Dong, 2006; Ren et al., 2010), triblock copolymers and poly(ester-urethane)s based on PCL-PLA block copolymers (Navarro-Baena, Kenny \& Peponi, 2014b).

This paper aims to study how the chemical structure influences the crystal nature as well as the crystallization behavior of several diblock copolymers based on PCL and PLLA. In particular, our study intends to contribute to a better knowledge of the crystallization behavior of PCL- $b$-PLLA diblock copolymers, focusing attention on the effect of PLLA molecular weight and PLLA amount on the crystallization behavior of both blocks, PLLA and PCL. The selected diblock copolymers have been synthesized by ring opening polymerization of L-lactide by using as initiator the hydroxyl groups of PCL monol. Moreover, the L-lactide amount has been varied in order to obtain nine different PCL- $b$-PLLA diblock copolymers with different ratios between the two blocks. In particular, the crystalline parameters for both PCL and PLLA crystals were determined by using wide-angle X-ray diffraction (WAXD) analysis and the crystallite size through the application of the Scherrer equation. The melting behavior of the copolymers was studied by small-angle X-ray scattering (SAXS) experiments performed in a synchrotron facility, by studying the variation of the long period with the temperature and by studying the correlation function. Finally, the crystallization behavior from the melt was investigated by calorimetry following the Avrami approach. Hence, the parameters of the crystallinity nucleation and growth as well as the energy involved during the crystallization have been obtained.

\section{Materials and methods}

\subsection{Materials}

L-lactide (L-LA), $\varepsilon$-caprolactone ( $\varepsilon$-CL) and tin(II) 2-ethylhexanoate were purchased from Sigma Aldrich. All the materials were used without further purification.

\subsection{Synthesis}

The diblock copolymers were synthesized by ring opening polymerization of L-LA using the same procedure as described in our previous work (Peponi et al., 2012). Thus, firstly the synthesis of PCL-OH and PLLA-OH was performed by using 1-butanol as initiator for the ring opening polymerization of $\varepsilon$-CL and L-LA, respectively. PCL-OH with a molecular weight of about $5000 \mathrm{~g} \mathrm{~mol}^{-1}$ was used for the further synthesis of diblock copolymers and taken as the reference sample $\mathrm{PCL}_{100}-b$-PLLA ${ }_{0}$, while PLLA-OH is the reference sample $\mathrm{PCL}_{0}-b$-PLLA ${ }_{100}$. We chose to use a PCL block with constant molecular weight in order to compare the influence of the PLLA block on the crystalline behavior of the PCL block. Then, using the synthesized PCL-OH, the reaction of diblock copolymers was performed by ring opening polymerization of L-LA using the hydroxyl end group of PCL-OH as initiator. All the reactions were carried out in bulk. After the reaction of PCL-OH, PLLA-OH and the PCL- $b$-PLLA diblock copolymers, the products were dissolved in chloroform, precipitated in cold methanol and filtered off in order to remove the non-reacted monomer. Finally, all the materials were dried under high vacuum for $24 \mathrm{~h}$ prior to characterization. Nine materials with different PCL-PLLA block ratios were studied, that is $0-100,15-85,20-80,30-70,50-50,70-30,80-20,85-15$, 100-0. The PCL molecular weight is constant at about $5000 \mathrm{~g} \mathrm{~mol}^{-1}$ for all the PCL- $b$-PLLA diblock copolymers and only the PLLA molecular weight varies, as indicated in Table 1. 
Table 1

Main chemical characteristics of the PCL- $b$-PLLA diblock copolymers studied.

\begin{tabular}{lccccc}
\hline Sample & $\%$ PCL $\dagger$ & $\%$ PLLA $\dagger$ & $\%$ PCL $\ddagger$ & $\%$ PLLA $\neq$ & Mn PLLA $\ddagger$ \\
\hline PCL-OH & 100 & 0 & 100 & 0 & - \\
P1 & 85 & 15 & 91 & 9 & 606 \\
P2 & 80 & 20 & 79.6 & 20.4 & 1696 \\
P3 & 70 & 30 & 76.7 & 23.3 & 1872 \\
P4 & 50 & 50 & 52.3 & 47.7 & 5918 \\
P5 & 30 & 70 & 29.5 & 70.5 & 13488 \\
P6 & 20 & 80 & 21.4 & 78.6 & 17310 \\
P7 & 15 & 85 & 14.6 & 85.4 & 27069 \\
PLLA-OH & 0 & 100 & 0 & 100 & 7680 \\
\hline
\end{tabular}

$\dagger$ Theoretical values. $\$$ Values obtained from ${ }^{1} \mathrm{H}$ NMR analysis.

\subsection{Nuclear magnetic resonance}

The chemical structure was investigated with ${ }^{1} \mathrm{H}$ NMR using a Varian Mercury Plus NMR $400 \mathrm{MHz}$ apparatus. Samples of $10 \mathrm{mg}$ were dissolved in deuterated chloroform, and the solution was filtered with a cotton filter before the analysis. A total of 32 spectra were recorded. The molecular structures of PCL-OH, PLLA-OH and PCL- $b$-PLLA diblock copolymers were studied, taking into account the work of Bero et al. (1993).

\subsection{X-ray characterization}

WAXD measurements were performed using a Bruker D8 Advance instrument with a $\mathrm{Cu} K \alpha$ source $(0.154 \mathrm{~nm})$ and a Vantec1 detector. Three spectra were recorded for each material. The interplanar distance $\left(d_{h k l}\right)$ was calculated considering the orthorhombic unit cell for both PCL and PLLA (Bittiger et al., 1970; Alemán et al., 2001). The crystal size in the $(h k l)$ direction was calculated through the application of the Scherrer equation:

$$
D=\frac{k d}{\beta \cos \theta},
$$

where $D$ is the crystal size, $k$ is the shape factor with a typical value of 0.9 (Navarro-Baena, Kenny \& Peponi, 2014b), $\lambda$ is the wavelength of the incident wave, $\beta$ is the broadening of the peak at half the maximum peak and $\theta$ is the diffraction angle. For the measurements at different temperatures a thermally controlled chamber was used.

SAXS measurements were taken at the beamline BM16 of the European Synchrotron Radiation Facility (Grenoble, France). Samples were melted and sealed in aluminium pans and placed inside a Linkam hot stage. The samples were heated at $10 \mathrm{~K} \mathrm{~min}^{-1}$ while the SAXS spectra were recorded. Calibration of temperature gave a difference of approximately $7 \mathrm{~K}$ between the temperature at the Linkam and the real temperature of the sample. Detector calibration was done with silver behenate and the long period was determined as $2 \pi / q(q$ is the magnitude of the scattering vector). The correlation function was determined using the following equation:

$$
\gamma(r)=(1 / Q) \int I(q)^{2} q \cos (q r) \mathrm{d} q,
$$

where $\gamma(r)$ is the correlation function, $r$ represents the distance in real space, $Q$ is the invariant, $q$ is the scattering vector magnitude and $I(q)$ is the intensity. For the extrapolation of the tail $(q=\infty)$ a Porod function was used, while for the back extrapolation $(q=0)$ a Guinier function was employed.

\subsection{Differential scanning calorimetry characterization}

The dynamic and isothermal differential scanning calorimetry (DSC) measurements were performed in a Perkin Elmer DSC 8500 instrument. Three cycles were programmed for the dynamic measurements; first, heating scans (from 298 to $473 \mathrm{~K}$ ), then a cooling scan (from 473 to $183 \mathrm{~K}$ ) and a final heating scan (from 183 to $473 \mathrm{~K}$ ). The heating/cooling rate for every scan was $10 \mathrm{~K} \mathrm{~min}^{-1}$. For the isothermal measurements the working temperatures were chosen taking into account the results obtained in the dynamic DSC experiments. The experimental data were fitted with the Avrami equation:

$$
X(t)=\frac{X_{\mathrm{c}}(t)}{X_{\mathrm{t}}}=\frac{\int_{t_{0}}^{t}\left(\mathrm{~d} H_{\mathrm{c}} / \mathrm{d} T\right) \mathrm{d} T}{\int_{t_{0}}^{t_{\infty}}\left(\mathrm{d} H_{\mathrm{c}} / \mathrm{d} T\right) \mathrm{d} T}=1-\exp \left(-K t^{n}\right),
$$

where $X(t)$ is the relative crystallinity, $X_{\mathrm{c}}(t)$ is the degree of crystallinity at time $t, X_{\mathrm{t}}$ is the crystallinity reached at the end of the crystallization process, $H_{\mathrm{c}}$ is the melting enthalpy, $T$ is the temperature, $K$ is a constant related to the crystallization kinetics, nucleation and growth rate, and $n$ is the Avrami index, related to the type of nucleation and the growth process (Banks \& Sharples, 1963).

Different authors have studied the temperature dependence of the kinetic constant $(K)$ by the assumption that this constant is thermally activated, so it can be represented by an Arrhenius equation (Kemmish \& Hay, 1985; Yang et al., 2006; Kenny \& Maffezzoli, 1991; Cebe \& Hong, 1986). Using this assumption, it is possible to obtain the activation energy of the growth and nucleation process by fitting the value of the $K$ constant at different temperatures with the Arrhenius equation as follows:

$$
K^{1 / n}=k_{0} \exp \left(-\frac{\Delta E}{R_{0} T_{\mathrm{c}}}\right) \Rightarrow \frac{1}{n} \ln K=\ln k_{0}-\frac{\Delta E}{R_{0} T_{\mathrm{c}}},
$$

where $k_{0}$ is a temperature-independent constant, $n$ is the Avrami index, $R_{0}$ is the gas constant with a typical value of $8.314 \mathrm{~J} \mathrm{~mol}^{-1} \mathrm{~K}^{-1}, \quad T_{\mathrm{c}}$ is the crystallization temperature expressed in kelvin and $\Delta E$ is the activation energy.

\section{Results and discussion}

\subsection{Synthesis}

The molecular weight of the synthesized PCL- $b$-PLLA diblock copolymer was determined by ${ }^{1} \mathrm{H}$ NMR. The values were obtained through comparison of the signals of the methine proton of polymerized lactide (at approximately 5.25 p.p.m.) and the proton of polymerized $\varepsilon$-caprolactone (at approximately 4.05 p.p.m.), as was already reported for PCL$b$-PLLA diblock copolymers (Peponi et al., 2012). The 
experimental values of the amount of each block reported in Table 1 are in good agreement with the theoretical ones, indicating that the reactions are well performed.

\subsection{X-ray diffraction analysis}

First, WAXD analysis was performed to check the crystalline nature of the synthesized PCL- $b$-PLLA diblock copolymers after the synthesis procedure, without performing further thermal treatments. The crystalline unit cell of PCL is shown in Fig. 1. This representation, according to the work of Bittiger et al. (1970), corresponds to a primitive orthorhombic cell having two polymer chains with opposite orientation into the unit cell, and the space group is $P 2_{1} 2_{1} 2_{1}$. Furthermore, for these materials PLLA also crystallizes in an orthorhombic cell with a similar packing mode to PCL, $P 2_{1} 2_{1} 2_{1}$ (Alemán et al., 2001).

The WAXD diffraction patterns for the different PCL- $b$ PLLA diblock copolymers are shown in Fig. 2. The spectra present the main characteristic diffraction peaks (marked with a dashed line) corresponding to both the PCL and PLLA blocks. These values are in good agreement with the data reported in the literature (Brizzolara et al., 1996).

The more intense diffraction peaks of PLLA, 200/110 and 203 , were found for copolymers having more than $20 \%$ of PLLA content. In addition, the most characteristic diffraction peaks of PCL, 110, 111 and 200, were observed for copolymers with more than $20 \%$ of PCL content. These main peaks were used for further determination of parameters. However, the parameters were only determined for copolymers with more than $30 \%$ of PCL or PLLA, respectively, to avoid introducing errors due to the weak signals. Regarding Fig. 2, it can be noted that the PLLA peaks do not vary with the composition. In fact, the PCL- $b$-PLLA diblock copolymer peaks match with the dashed line corresponding to PLLA. Conversely, the peaks corresponding to the PCL block in the PCL- $b$-PLLA diblock copolymers do not match with the dashed lines, and they are shifted depending on the composition. This fact indicates that the presence of PLLA interferes with and modifies the crystalline unit cell of PCL.

In Fig. 2 the WAXD spectra for all the samples studied are reported. The interplanar distances obtained from Fig. 2 are

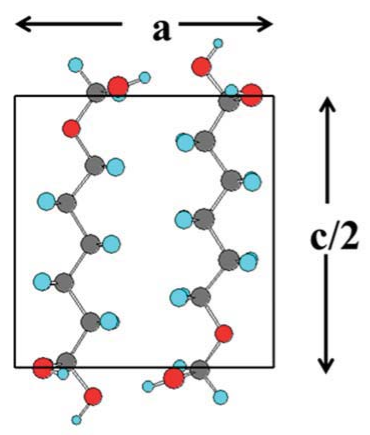

ac projection

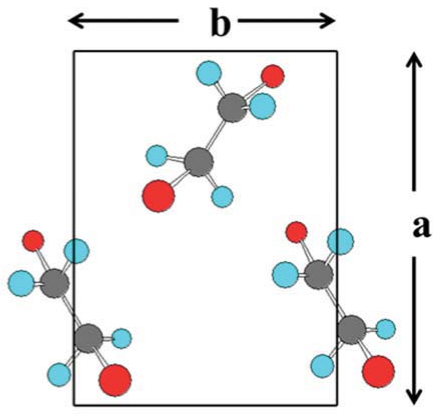

ab projection
Figure 1

PCL unit cell: $a c$ projection (fiber axis) and $a b$ projection (basis).
Table 2

Interplanar distances corresponding to the diffraction peaks observed for the different polymers.

\begin{tabular}{llllllllll}
\hline$h k l$ & PCL-OH & P1 & P2 & P3 & P4 & P5 & P6 & P7 & PLLA-OH \\
\hline 110 & 4.13 & 4.17 & 4.15 & 4.16 & 4.16 & 4.15 & - & - & - \\
111 & 4.01 & 4.06 & 4.04 & 4.05 & 4.03 & 3.99 & - & - & - \\
200 & 3.73 & 3.76 & 3.75 & 3.75 & 3.75 & 3.75 & - & - & - \\
$200 / 100$ & - & - & - & 5.32 & 5.31 & 5.31 & 5.31 & 5.31 & 5.30 \\
203 & - & - & - & 4.65 & 4.64 & 4.65 & 4.66 & 4.66 & 4.67 \\
\hline
\end{tabular}

summarized in Table 2, while the crystal lattice parameters calculated from the interplanar distances are reported in Table 3 .

It is easy to note that the $d_{h k l}$ values for $\mathrm{P} 1$ are the highest ones, higher than those obtained for PCL-OH as well as for the other copolymers. This behavior is related to the curves shown in Fig. 2 and the $c$ values reported in Table 3. It can be observed that the $c$ parameter first increased (P1) and then decreased owing to the presence of PLLA chains. This fact suggests that the increase of crystalline PLLA in the copolymer can promote the nucleation of a large number of smaller PCL crystals but, in the case of the smaller PLLA concentration (P1), this is not necessarily true. It has been already demonstrated that at this small concentration (15\%) PLLA is not able to crystallize, and thus it is not able to nucleate the PCL crystallization. Therefore, the only effect of the presence of the PLLA chains on the PCL crystal is a hindrance of PCL crystallization, leading to a higher PCL crystal dimension.

The lattice parameters, summarized in Table 3 , have been calculated by using equation (5), thus taking into account that both PCL and PLLA crystallize in the orthorhombic cell form:

$$
\frac{1}{d_{h k l}^{2}}=\frac{h^{2}}{a^{2}}+\frac{k^{2}}{b^{2}}+\frac{l^{2}}{c^{2}} \text {. }
$$

The values for the lattice parameters are in good agreement with the data found in the literature (Bittiger et al., 1970; Brizzolara et al., 1996; De Santis \& Kovacs, 1968). The basis lattice parameters $(a$ and $b$ ) of PCL are around $7.50 \AA$ for $a$

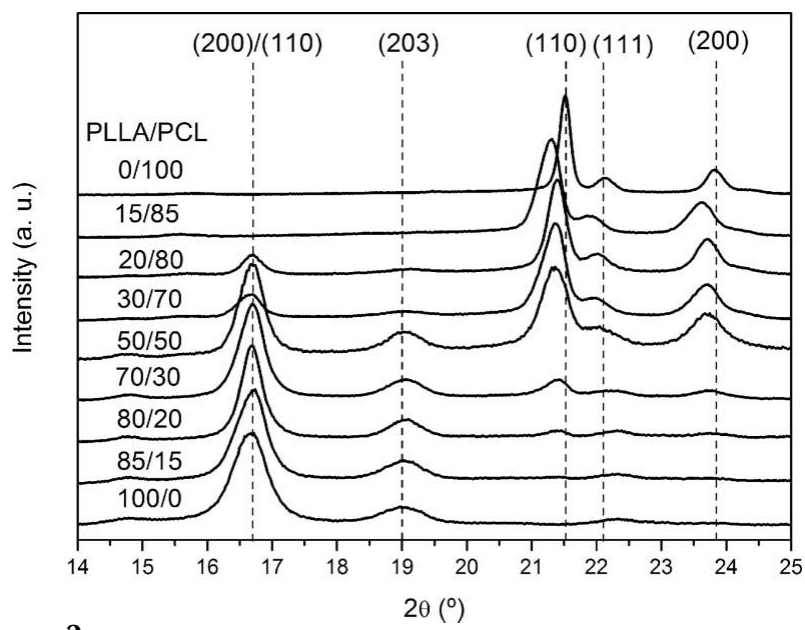

Figure 2

WAXD spectra for PCL- $b$-PLLA diblock copolymers. The dashed lines correspond to the more intense peaks for both PLLA and PCL. Their corresponding planes are also indicated. 
Table 3

Crystalline data obtained from WAXD characterization.

Values in parentheses are the error on the least significant digits.

\begin{tabular}{|c|c|c|c|c|c|c|c|c|c|c|}
\hline \multirow[b]{2}{*}{ Sample } & \multicolumn{5}{|c|}{ PCL crystalline parameters } & \multicolumn{5}{|c|}{ PLLA crystalline parameters } \\
\hline & $a$ & $(\AA)$ & $\begin{array}{l}c \\
(\AA)\end{array}$ & $\begin{array}{l}\text { Crystal size } \\
\left(L_{110}\right)(\mathrm{nm})\end{array}$ & $X_{\mathrm{c}}(\%)$ & $a$ & $\begin{array}{l}b \\
(\AA)\end{array}$ & $\begin{array}{l}c \\
(\AA)\end{array}$ & $\begin{array}{l}\text { Crystal size } \\
\left(L_{200 / 110}\right)(\mathrm{nm})\end{array}$ & $X_{\mathrm{c}}(\%)$ \\
\hline PCL-OH & 7.46 & 4.95 & 17.26 & $79.6(22)$ & $59.1(24)$ & - & - & - & - & - \\
\hline P1 & 7.53 & 5.01 & 17.73 & 41.2 (16) & $68.1(26)$ & - & - & - & - & - \\
\hline P2 & 7.50 & 4.98 & 17.34 & $47.5(18)$ & $67.1(26)$ & - & - & - & - & - \\
\hline P3 & 7.50 & 4.99 & 17.62 & 37.9 (19) & $63.8(25)$ & 10.64 & 6.14 & 28.85 & $18.5(12)$ & $49.1(16)$ \\
\hline P4 & 7.50 & 4.99 & 16.23 & $32.8(23)$ & $63.1(25)$ & 10.62 & 6.13 & 28.61 & 36.1 (19) & $65.1(26)$ \\
\hline P5 & 7.49 & 4.98 & 14.53 & $35.9(24)$ & $78.0(31)$ & 10.61 & 6.13 & 29.05 & 35.5 (14) & $66.1(26)$ \\
\hline P6 & - & - & - & - & - & 10.62 & 6.13 & 29.05 & 41.9 (15) & $68.7(26)$ \\
\hline P7 & - & - & - & - & - & 10.61 & 6.13 & 29.17 & $30.4(12)$ & $58.8(24)$ \\
\hline PLLA-OH & - & - & - & - & - & 10.60 & 6.12 & 29.53 & 24.9 (11) & $57.7(23)$ \\
\hline
\end{tabular}

crystallinity for the PLLA block is higher for intermediate compositions (50-80 wt\% PLLA) than for extreme compositions. This result suggests that neat PLLA forms many small crystallites and, by increasing the PCL content, the nucleation is reduced, leading to bigger crystallites. For the copolymer with $30 \mathrm{wt} \%$ PLLA the crystal size and the degree of crystallization are also reduced because of the presence of PCL chains. So, the presence of each block modifies the crystal nature of the other and, in particular for PCL, the variations are more important taking into account that all the diblock copolymers were synthesized starting from PCL-OH with the same molecular weight.

In order to perform an in depth study on the double-crystalline nature and melting behavior of the PCL- $b$-PLLA diblock copolymers, SAXS experiments were performed. The SAXS spectra were recorded at different temperatures, to visualize the effects of the thermal transitions. In Fig. 4, the spectra corresponding to the sample P5, with a content of $70 \mathrm{wt} \%$ of PLLA, are reported. The peak that appears in these spectra reflects the phase separation between the lamellae and the amorphous regions, which have different electron densities. It can be noted that the peak changes on increasing the temperature owing to the melting of the PCL block. After that, at higher temperatures, the peak disappears because of the melting of PLLA, leaving a homogeneous melt. For the other PCL- $b$-PLLA diblock copolymers (data not shown), similar spectra were collected.

The long period was determined from the collected spectra at each temperature by applying the Bragg equation to the $q$ values corresponding to the maximum of intensity. In Fig. 5, the values of long period at different temperatures for the six copolymers are represented. All PCL- $b$-PLLA diblock copolymers exhibit the same trend. First, the spectrum remains constant with the increase of temperature. Then, at a certain temperature, PCL crystals melt, increasing the long data obtained show a completely different trend. In fact, the maximum value for both the crystal size and the degree of

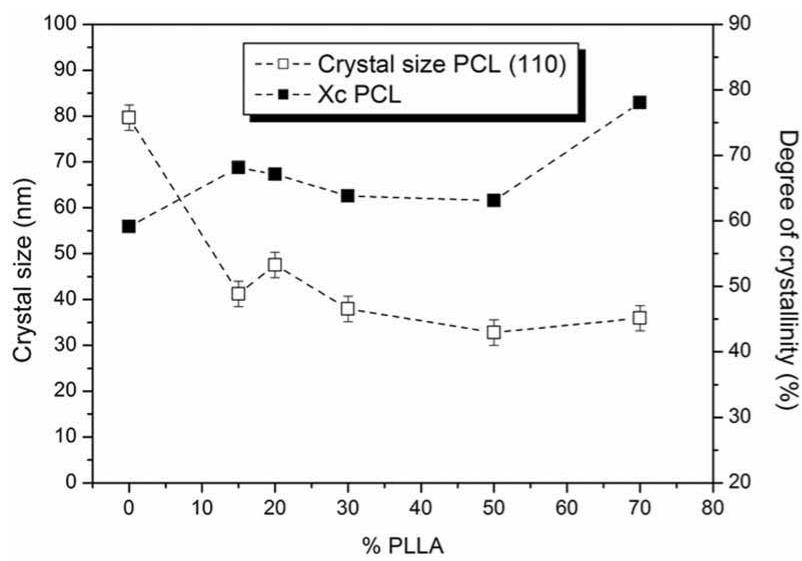

(a)

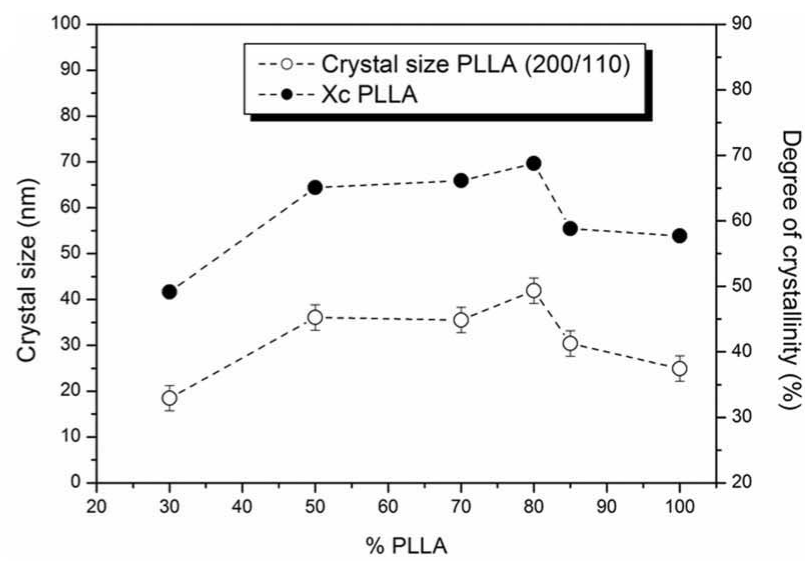

(b)

Figure 3

Crystallite size obtained using the Scherrer equation and the degree of crystallinity as a function of PLLA content in the diblock copolymer for ( $a$ ) the PCL block and $(b)$ the PLLA block. 
period value. Finally, the long period value increases and tends to infinity owing to the melting of PLLA, indicating that a homogeneous melt was achieved. The sample P1 $\left(\mathrm{PCL}_{85}-b\right.$ $\mathrm{PLLA}_{15}$ ) (filled squares) only presents the PCL as a crystalline

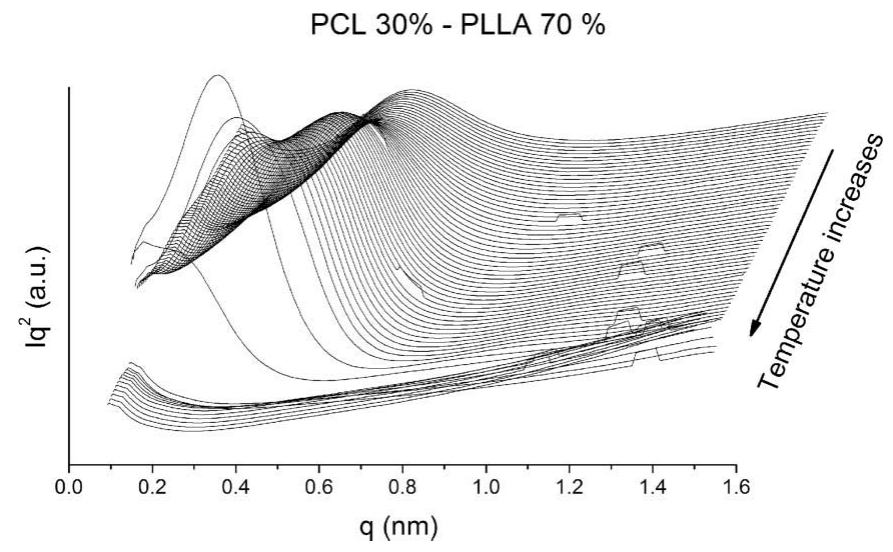

Figure 4

SAXS spectra corresponding to sample P5 $\left(\mathrm{PCL}_{30}-b-\mathrm{PLLA}_{70}\right)$.

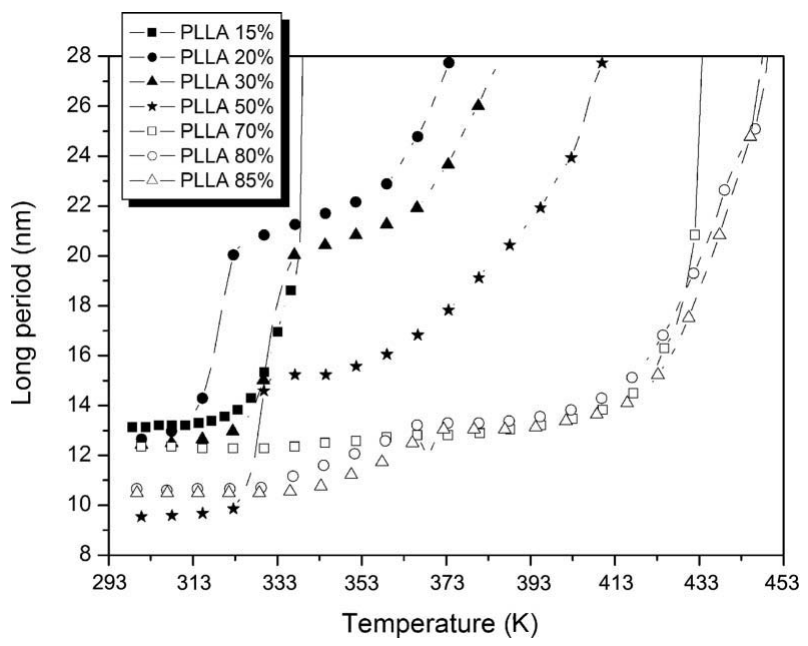

Figure 5

Long period values versus temperature for the PCL-b-PLLA diblock coploymers.

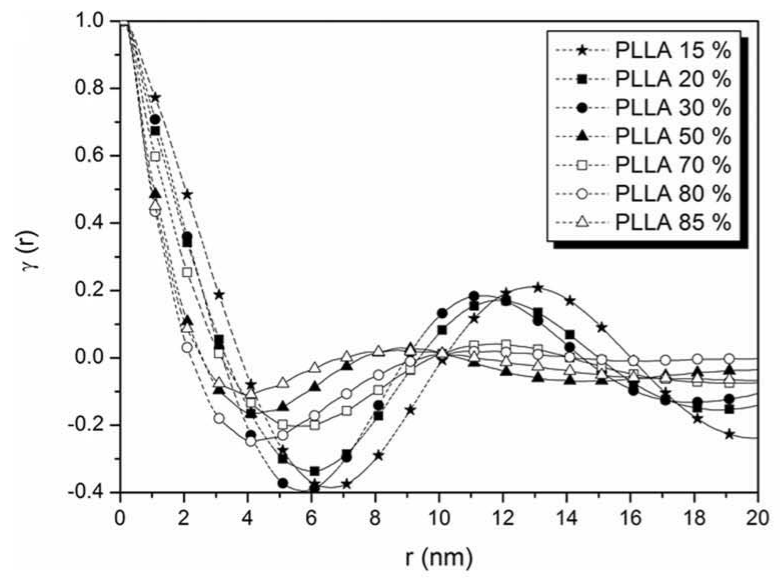

(a) block and the long period tends to infinity at $333 \mathrm{~K}$. The other diblock copolymers present both crystalline blocks. On one hand, the samples with low PLLA content (filled symbols for PLLA content $\leq 50 \%$ ) present large variations in the long period before and after PCL melting, reaching higher values than for the diblock copolymers rich in PLLA (unfilled symbols). A different behavior is found for the PLLA-rich materials. In fact, they show a constant value for the long period after PCL melting, around $13 \mathrm{~nm}$. Thus, for the diblock copolymer with high PCL content (up to $50 \mathrm{wt} \%$ ) the PCL amorphous chains affect the formation of the PLLA lamellae.

In Fig. 6, the one-dimensional correlation functions for all PCL- $b$-PLLA diblock copolymers at room temperature (Fig. $6 a$ ) and after the melting of the PCL block (Fig. 6b) are shown. This function is related to the electron density fluctuation due to the coexistence of two phases showing periodicity (Bernal et al., 2012). The position of the first peak corresponds to the long period value. It is worth noting that the values obtained from the study of the correlation function are slower than those obtained before using the Bragg equation. In fact, as is reported in the literature, the value obtained from the correlation function gives a more reliable value (Santa Cruz et al., 1991).

At room temperature (Fig. 6a), the long period values present the same trend as the values obtained from the Bragg equation (Fig. 5). The correlation function is significantly changed after the melting of the PCL block (Fig. 6b) for each composition. The long period, which is the first local maximum of the correlation function, was strongly shifted for the PCL- $b$ PLLA diblock copolymers with low PLLA content (filled symbols), while for those rich in PLLA (unfilled symbols), the function achieved a more defined shape, reaching a similar constant value for the long period.

\subsection{Isothermal crystallization analysis}

The crystallization temperatures for experimental DSC isothermal analysis were chosen for each block in the interval between their melting and the crystallization temperatures

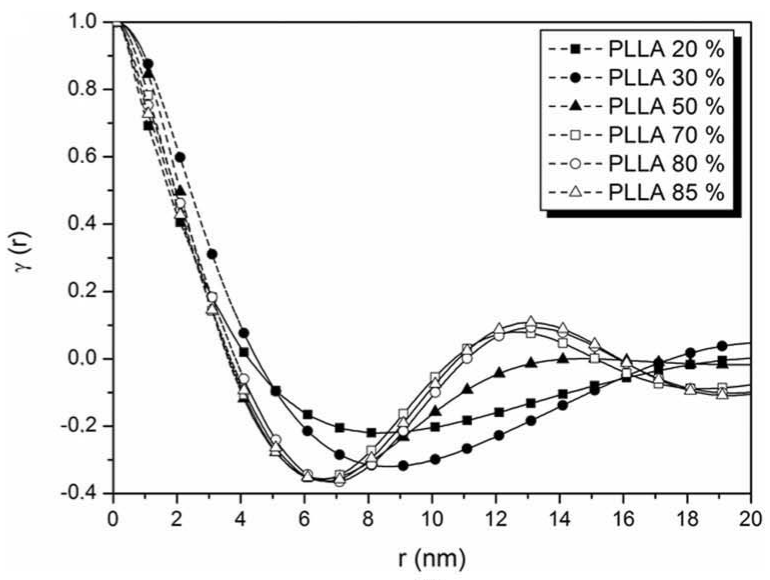

(b)

Figure 6

One-dimensional correlation function for the different diblock copolymers at room temperature $(a)$ and after PCL melting $(b)$. 
obtained in dynamic DSC experiments (Peponi et al., 2012). In particular the isothermal crystallization of the PLLA block was studied in the interval 367-409 K, while the PCL block isothermal characterization was performed in the interval 306-316 K. For a better understanding of the crystallization behavior, the isothermal DSC results have been divided into two parts depending on the block that crystallizes. First, the results for the PLLA block and then the results corresponding to the PCL block are reported and discussed.

3.3.1. PLLA isothermal crystallization results. In Fig. 7 the values related to the PLLA-OH experimental data (Fig. 7a) as

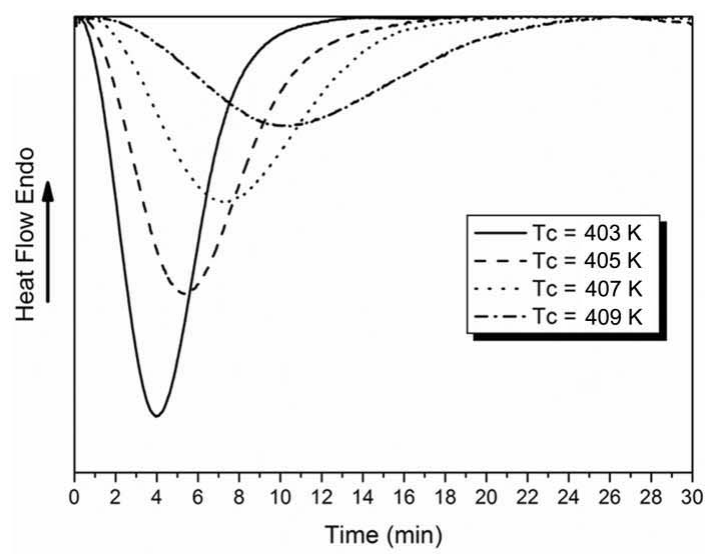

(a)

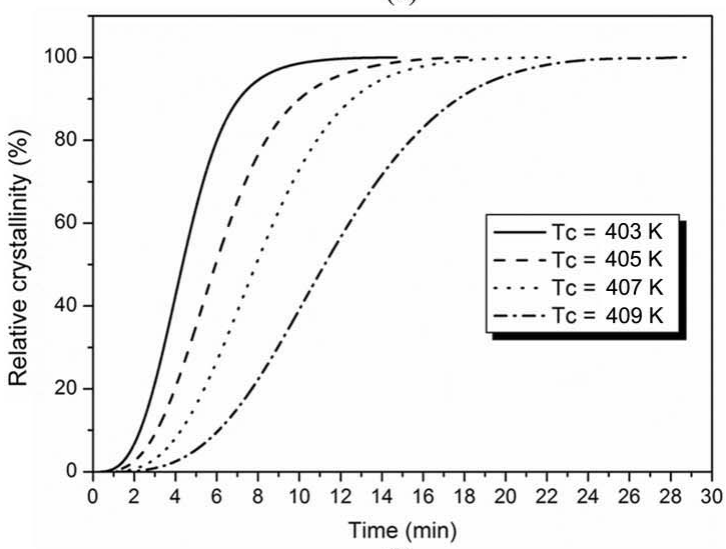

(b)

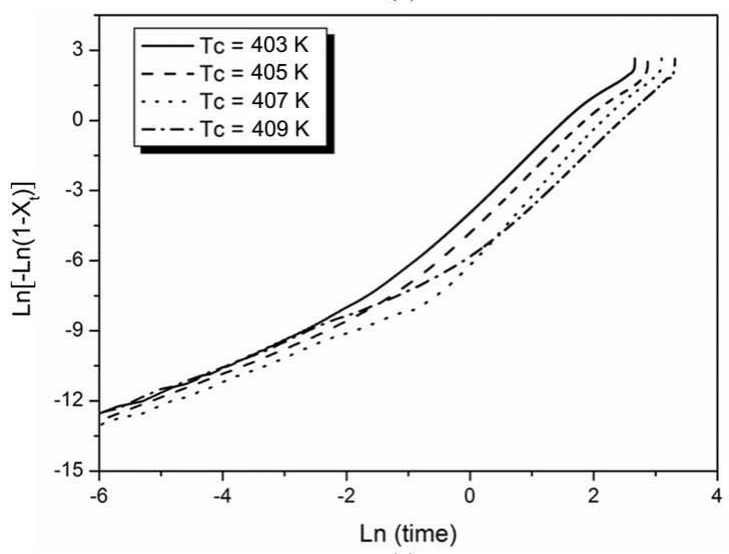

(c)

Figure 7

Isothermal DSC experiments for PLLA-OH: $(a)$ experimental data; $(b)$ relative crystallinity versus time; (c) Avrami fit.
Table 4

Isothermal crystallization parameters for the PLLA block.

\begin{tabular}{lccccc}
\hline Sample & $\%$ PLLA & $\Delta T_{\mathrm{c}}(\mathrm{K})$ & $n$ & $\ln K_{0}$ & $\Delta E\left(\mathrm{~J} \mathrm{~mol}^{-1}\right)$ \\
\hline PLLA-OH & 100 & $403-409$ & 2.64 & -63.4 & -207 \\
P7 & 85 & $388-403$ & 2.69 & -53.7 & -171 \\
P6 & 80 & $388-394$ & 2.55 & -96.2 & -308 \\
P5 & 70 & $378-393$ & 2.60 & -46.8 & -146 \\
P4 & 50 & $378-383$ & 2.51 & -86.8 & -268 \\
P3 & 30 & $367-373$ & 2.54 & -74.7 & -226 \\
\hline
\end{tabular}

well as the crystallinity conversion (Fig. 7b) and the Avrami curves for each crystallization temperature (Fig. 7c) are reported. For the other samples the data are not shown but they present similar behavior. The isothermal DSC experimental curves for the PLLA-OH sample are reported in Fig. 7(a), showing that when the crystallization temperatures increases the PLLA block needs more time to achieve complete crystallization.

The relative crystallinity (Fig. $7 b$ ) was determined using the experimental DSC curves (Fig. 7a). These data were fitted using the Avrami equation [equation (3)]; the Avrami fitted curves obtained at each crystallization temperature are shown in Fig. 7(c). To obtain the Avrami parameters, a linear fit was performed in the crystallization region from 3 to $20 \%$ of conversion. This region has been commonly used before for this kind of analysis (Navarro-Baena, Kenny \& Peponi, 2014b; Lorenzo et al., 2007).

In Table 4 , the results for the PCL- $b$-PLLA diblock copolymers after the isothermal crystallization analysis, in terms of PLLA content, crystallization temperature range $\left(\Delta T_{\mathrm{c}}\right)$, Avrami index $(n)$, logarithm of $K_{0}$ and the activation energy $(\Delta E)$ obtained after applying the Arrehnius equation, are summarized.

For all the samples, the Avrami index values are between 2.0 and 3.0, indicating a tendency for a two-dimensional crystallite growth. As reported in the literature, the values between 2.0 and 3.0 indicate a combination of instantaneous and sporadic nucleation (Hay, 1971). Moreover, these results indicate that the crystallization kinetics for PLLA are not influenced by the presence or by the quantity of PCL chains. These values are well related to the data reported in the scientific literature for the PLLA block in different diblock copolymers (Kim et al., 2001; Hamley et al., 2005; Müller et al., 2006; Michell et al., 2011; Yang et al., 2006). The energies of the crystallization process obtained from the Arrhenius fit are lower compared with other PCL-PLLA block copolymers, such as for the triblock copolymers PLLA-PCL-PLLA or the poly(esterurethane)s, for which the energies range from -164.5 to -95.4 and from -136.4 to $-80.9 \mathrm{~J} \mathrm{~mol}^{-1}$, respectively, as reported by us in a previous work (Navarro-Baena, Kenny \& Peponi, $2014 b$ ). This fact indicates that the diblock copolymer can more easily crystallize from the melt than more complex copolymers.

In Fig. 8, the crystallization half-times for the PCL- $b$-PLLA diblock copolymers at different temperatures are shown. These values were obtained as the time necessary to reach a $50 \%$ of the whole crystallization. This parameter represents 
the overall crystallization rate and it is well related to the constant $K$ determined from the Avrami analysis (Castillo et al., 2010). For each diblock copolymer studied, the reported values show the same trend, that is, the crystallization rate decreases when the crystallization temperature increases. The experimental points follow a hyperbolic curve. It seems that the curve shifts its position to lower values of crystallization temperature (so higher crystallization rates) when the PLLA content decreases. The neat PLLA shows the smallest crystallization rate compared with the other diblock copolymers. Therefore, it seems that the presence of PCL amorphous chains increases the crystallization rate of PLLA crystals.

3.3.2. PCL isothermal crystallization results. For the PCL crystallization studies, it was necessary to crystallize first the PLLA block to avoid crystallization effects of PLLA. Thus, the crystallization of PCL chains takes place in the presence of PLLA crystals. Fig. 9 summarizes the plots of the isothermal DSC experiments (Fig. 9a), the relative crystallinity (Fig. 9b) and the data fitted using the Avrami equation (Fig. 9c). As occurs for the PLLA crystallization, the crystallization rate of PCL decreases when the crystallization temperature increases.

The data obtained for the PCL isothermal crystallization are summarized in Table 5. Several differences were expected in the crystallization kinetics compared with PLLA, owing to the restriction imposed by the presence of PLLA crystals during the crystallization process of PCL as well as to the high rigidity of the frozen PLLA chains, which are at a temperature below their glass transition temperature.

For the PCL block, the Avrami index values are between 2.0 and 3.0, as occurs for the PLLA block, indicating that also in this case the crystallization has a two-dimensional growth. However, for PCL blocks a clear trend can be noted. On increasing the PLLA content in the diblock copolymers, the Avrami index values increase from 2.2 to 2.9 depending on the composition. Therefore, the neat PCL crystallizes with a sporadic nucleation, the crystalline nuclei appear randomly in time and then they grow. However, in the diblock copolymers, the addition of PLLA induces a predetermined nucleation of PCL, that is, all the nuclei are formed at the initial time.

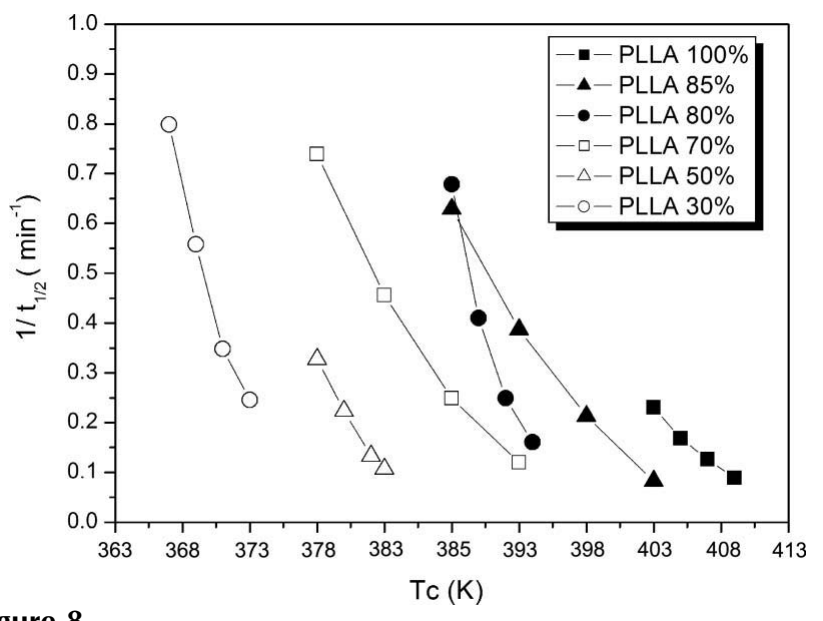

Figure 8

Inverse $t_{1 / 2}$ for PLLA crystals at different crystallzation temperatures.
Table 5

PCL isothermal crystallization parameters.

\begin{tabular}{lrrrrl}
\hline Sample & $\% \mathrm{PCL}$ & $\Delta T_{\mathrm{c}}(\mathrm{K})$ & $n$ & $\ln K_{0}$ & $\Delta E\left(\mathrm{~J} \mathrm{~mol}^{-1}\right)$ \\
\hline PCL-OH & 100 & $313-316$ & 2.24 & -113 & -292 \\
P1 & 85 & $310-313$ & 2.50 & -84.0 & -216 \\
P2 & 80 & $310-313$ & 2.46 & -44.6 & -115 \\
P3 & 70 & $310-313$ & 2.61 & -42.0 & -108 \\
P4 & 50 & $306-309$ & 2.70 & -44.1 & -110 \\
P5 & 30 & $306-309$ & 2.90 & -78.3 & -195 \\
\hline
\end{tabular}

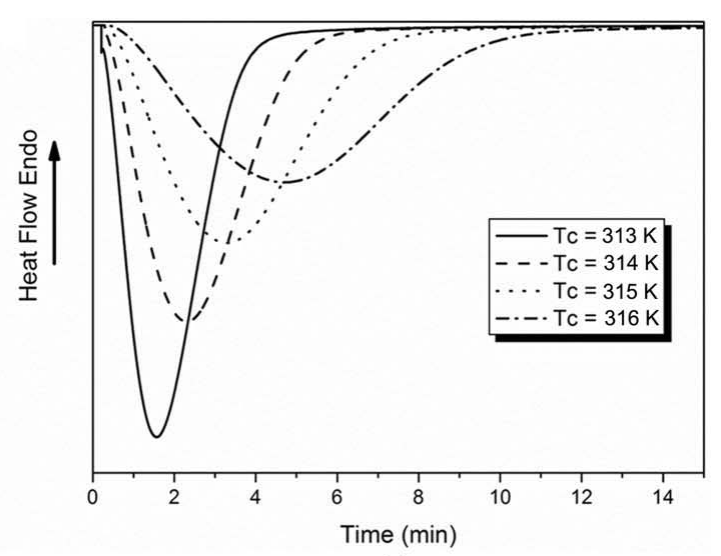

(a)

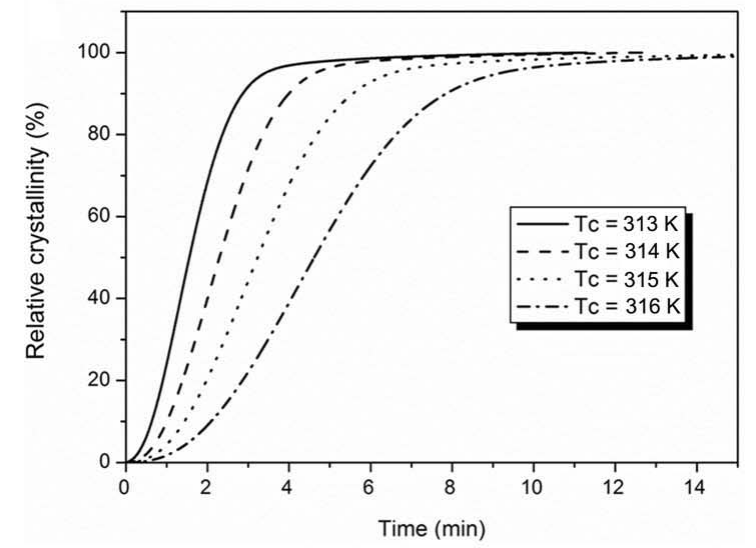

(b)

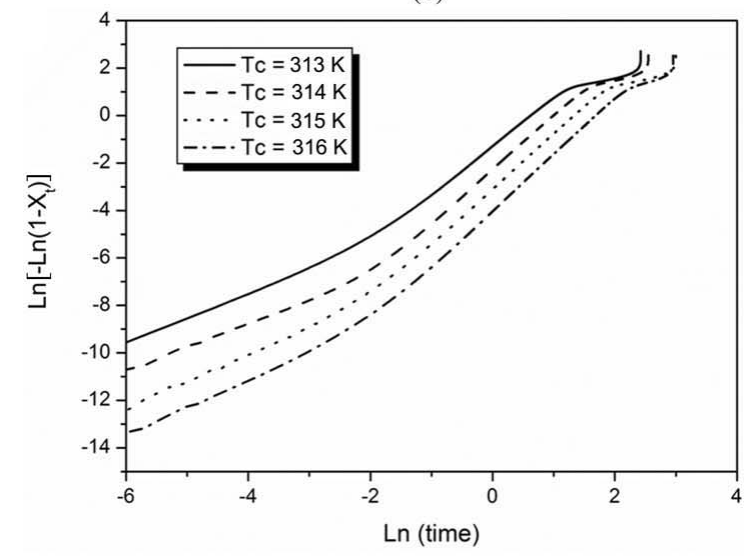

(c)

Figure 9

Isothermal DSC experiments for PCL-OH: (a) experimental data; $(b)$ relative crystallinity versus time; (c) Avrami fit. 


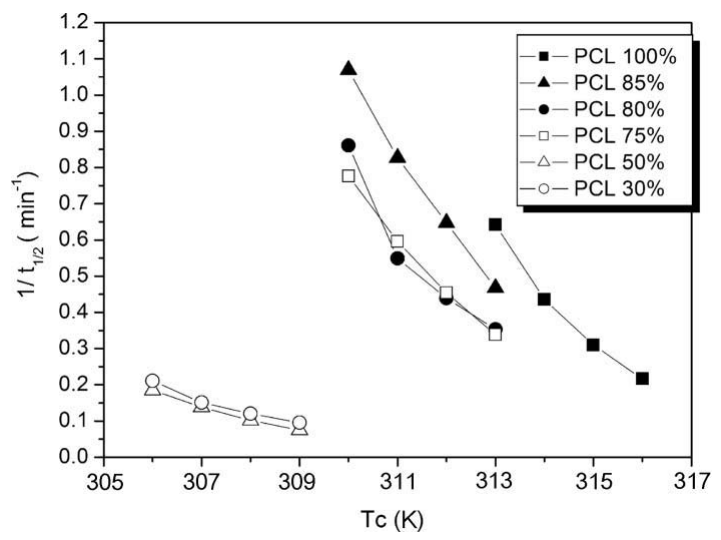

Figure 10

Inverse $t_{1 / 2}$ for PCL crystals at different crystallzation temperatures.

In contrast with this result, Castillo et al. (2010) reported a decrease in the Avrami index when the PCL content decreased for PCL-PLLA diblock copolymers. The explanation of this result might be referred to the polymer structure. The PCL- $b$-PLLA diblock copolymers presented here have the same PCL molecular weight, while those reported by Castillo and co-workers represented block copolymers with different PCL molecular weight and content.

In Fig. 10, the values of the inverse of crystallization halftime of PCL in the diblock copolymers are reported. As occurs for the PLLA block, for each copolymer this value decreased when the crystallization temperature increased. So, the crystallization rate was lower at higher crystallization temperatures, leading to a parabolic curve. It can be noted that for the crystallization of copolymers with a percentage of PCL below $50 \%$ ( $\mathrm{P} 4$ and $\mathrm{P} 5$, unfilled triangles and circles, respectively), the crystallization rate was very low compared to the higher PCL content copolymers. This fact reveals the confinement effect due to the relevant presence of PLLA crystals, which constrain the PCL chains to crystallize in a reduced space.

\section{Conclusions}

The crystalline nature, the melting behavior and the crystallization of several PCL- $b$-PLLA diblock copolymers have been studied, focusing the attention on the effect of the presence of one block on the behavior of the other. For this reason, PCL- $b$-PLLA diblock copolymers with different weight ratio between the two blocks $(0-100,15-85,20-80,30$ $70,50-50,70-30,80-20,85-15,100-0)$ were synthesized with approximately constant molecular weight of PCL and different PLLA lengths. To study the crystalline features, WAXD, SAXS and DSC analyses were carried out. All the synthesized PCL- $b$-PLLA diblock copolymers showed double-crystalline nature. The characterization of the crystalline unit cell of both the PCL and PLLA blocks was studied by WAXD analysis, determining the characteristic peaks of each one and the lattice parameters. The influence of copolymerization in the crystal unit cell for each crystalline block was discussed. In particular, the presence of PLLA increased the nucleation of PCL and prevented the growth of PCL crystallites, while when increasing the PCL content, the nucleation of PLLA was favored and the crystallites reached fewer dimensions. SAXS measurements confirmed the double-crystalline nature, and the long period obtained revealed the effect of PCL content on the lamellar structure of PLLA, finding for the PCL- $b$-PLLA diblock copolymers with high PCL content an increase of the long period after PCL melting. Furthermore, by an analysis of the correlation function, the coexistence of two phases in the PCL- $b$-PLLA diblock copolymers was confirmed.

The crystallization of each block from the melt at four different crystallization temperatures was studied by isothermal DSC measurements. The data were studied in terms of the Avrami equation, obtaining the Avrami exponent, the constant $K$ and the activation energies of the crystallization process. From these analyses, it was concluded that the Avrami indexes for both PLLA and PCL are between 2.0 and 3.0, suggesting a two-dimensional crystallization. Furthermore, different behaviors for PCL and PLLA were detected. The experimental data showed that, on increasing the PLLA content, the PCL nucleation became predetermined. Thus the PLLA crystals acted as nucleation agent, while for PLLA crystallization no trend was detected. For PLLA, the crystallization rates also increased when the PCL content increased, while the PLLA crystallization temperatures decreased when the PCL content increased owing to both the presence of amorphous PCL chains and the decrease of the PLLA molecular weight. Conversely, the values of the overall crystallization rate obtained for PCL demonstrated a maximum of crystallization rate for $\mathrm{PCL}_{85}-b$-PLLA 15 , while the crystallization rate decreased strongly for lower PCL contents. Therefore, these results reflect the influence of the confinement phenomena produced by the presence of PLLA crystals during the PCL crystallization.

We are indebted to the Spanish Ministry of Science and Innovation for their economic support of this research (MAT2013-48059-C2-1-R). LP acknowledges also the support of a JAEdoc grant from CSIC cofinanced by FSE. We are grateful to Dr Ana Labrador and Dr François Fauth for their help at BM16 station at the European Synchrotron Radiation Facility in Grenoble (France).

\section{References}

Albano, C., Papa, J., Baré, W. \& González, J. (2007). Rev. Fac. Ing. $U C V, 22,71-77$.

Alemán, C., Lotz, B. \& Puiggali, J. (2001). Macromolecules, 34, 47954801.

Alvarez, V. A., Kenny, J. M. \& Vázquez, A. (2003). J. Appl. Polym. Sci. 89, 1071-1077.

Banks, W. \& Sharples, A. (1963). Makromol. Chem. 59, 233-236.

Bernal, M. M., Martin-Gallego, M., Romasanta, L. J., Mortamet, A.-C., López-Manchado, M. A., Ryan, A. J. \& Verdejo, R. (2012). Polymer, 53, 4025-4032.

Bero, M., Kasperczyk, J. \& Adamus, G. (1993). Makromol. Chem. 194, 907-912.

Bittiger, H., Marchessault, R. H. \& Niegisch, W. D. (1970). Acta Cryst. B26, 1923-1927. 
Brizzolara, D., Cantow, H.-J., Diederichs, K., Keller, E. \& Domb, A. J. (1996). Macromolecules, 29, 191-197.

Castillo, R. V., Müller, A. J., Raquez, J. M. \& Dubois, P. (2010). Macromolecules, 43, 4149-4160.

Cebe, P. \& Hong, S.-D. (1986). Polymer, 27, 1183-1192.

De Santis, P. \& Kovacs, A. J. (1968). Biopolymers, 6, 299-306.

Di Lorenzo, M. L. (2005). Eur. Polym. J. 41, 569-575.

Hamley, I. W., Castelletto, V., Castillo, R. V., Müller, A. J., Martin, C. M., Pollet, E. \& Dubois, P. (2005). Macromolecules, 38, 463-472.

Hamley, I. W., Parras, P., Castelletto, V., Castillo, R. V., Müller, A. J., Pollet, E., Dubois, P. \& Martin, C. M. (2006). Macromol. Chem. Phys. 207, 941-953.

Hay, J. N. (1971). Brit. Polym. J. 3, 74-82.

Kemmish, D. J. \& Hay, J. N. (1985). Polymer, 26, 905-912.

Kenny, J. M. \& Maffezzoli, A. (1991). Polym. Eng. Sci. 31, 607-614.

Kim, J. K., Park, D.-J., Lee, M.-S. \& Ihn, K. J. (2001). Polymer, 42, 7429-7441.

Lambrigger, M. (1996). Polym. Eng. Sci. 36, 98-99.

Loo, Y.-L., Register, R. A., Ryan, A. J. \& Dee, G. T. (2001). Macromolecules, 34, 8968-8977.

Lorenzo, A. T., Arnal, M. L., Albuerne, J. \& Müller, A. J. (2007). Polym. Test. 26, 222-231.

Michell, R. M., Müller, A. J., Spasova, M., Dubois, P., Burattini, S., Greenland, B. W. \& Hamley, I. W. (2011). J. Polym. Sci. Part B Polym. Phys. 49, 1397-1409.

Müller, A. J., Castillo, R. V. \& Hillmyer, M. (2006). Macromol. Symp. 242, 17-181.

Navarro-Baena, I., Kenny, J. M. \& Peponi, L. (2014a). Cellulose. doi:10.1007/s10570-014-0446-5.
Navarro-Baena, I., Kenny, J. M. \& Peponi, L. (2014b). Polym. Degrad. Stab. 108, 140-150.

Navarro-Baena, I., Marcos-Fernández, A., Fernández-Torres, A., Kenny, J. M. \& Peponi, L. (2014). RSC Adv. 4, 8510-8524.

Pan, P., Kai, W., Zhu, B., Dong, T. \& Inoue, Y. (2007). Macromolecules, 40, 6898-6905.

Panine, P., Urban, V., Boesecke, P. \& Narayanan, T. (2003). J. Appl. Cryst. 36, 991-994.

Peponi, L., Navarro-Baena, I., Báez, J. E., Kenny, J. M. \& MarcosFernández, A. (2012). Polymer, 53, 4561-4568.

Peponi, L., Navarro-Baena, I., Sonseca, A., Gimenez, E., MarcosFernandez, A. \& Kenny, J. M. (2013). Eur. Polym. J. 49, 893-903.

Rahaman, M. H. \& Tsuji, H. (2013). J. Appl. Polym. Sci. 129, $2502-$ 2517.

Ren, J., Zhang, Z., Feng, Y., Li, J. \& Yuan, W. (2010). J. Appl. Polym. Sci. 118, 2650-2658.

Santa Cruz, C., Stribeck, N., Zachmann, H. G. \& Balta Calleja, F. J. (1991). Macromolecules, 24, 5980-5990.

Sarasua, J.-R., Prud'homme, R. E., Wisniewski, M., Le Borgne, A. \& Spassky, N. (1998). Macromolecules, 31, 3895-3905.

Sonseca, A., Peponi, L., Sahuquillo, O., Kenny, J. M. \& Giménez, E. (2012). Polym. Degrad. Stab. 97, 2052-2059.

Supaphol, P. (2001). Thermochim. Acta, 370, 37-48.

Wang, J.-L. \& Dong, C.-M. (2006). Macromol. Chem. Phys. 207, 554562.

Wasanasuk, K. \& Tashiro, K. (2011). Macromolecules, 44, 96509660.

Yang, J., Zhao, T., Liu, L., Zhou, Y., Li, G., Zhou, E. \& Chen, X. (2006). Polym. J. 38, 1251-1257. 